\title{
The legal duty of candour in healthcare: the lessons of history?
}

\author{
Dr Catherine Kelly* ANd Dr Oliver QuicK**
}

Centre for Health, Law, and Society, University of Bristol Law School

\begin{abstract}
Providers of health and social care in England are under a statutory duty to be open and honest with patients who suffer harm when receiving care or treatment. This 'duty of candour' was introduced by regulation 20 of the Health and Social Care Act 2008 (Regulated Activities) Regulations 2014 and is one of 13 fundamental standards of care regulated by the Care Quality Commission (CQC). This was hailed as a landmark for openness in patient-professional relationships and as having the potential for enhancing a safety culture in healthcare. However, the decision to supplement existing ethical duties and policy initiatives encouraging openness with a statutory duty was contentious and encountered considerable medical resistance. This paper will trace the background to the legal duty, analyse its contents and consider its enforcement and potential obstacles to its effectiveness. Our analysis will foreground resistance based in practitioners' and healthcare institutions' fear of litigation and prosecution in the UK. However, opposition to candour emerged within the medical profession prior to the emergence of modern liability systems. This paper will argue that in order to create a culture of candour it is important to look beyond the more commonly identified professional concerns about litigation and understand these historical trends. In particular, we argue that a longer-term understanding of medical resistance to openness has important lessons for the likely effectiveness of the legal duty of candour.
\end{abstract}

Keywords: law; history; candour; patient safety; professionalism; regulation.

\section{Introduction}

Tn November 2014, England introduced a statutory duty of candour on healthcare 1 providers to be open and honest with patients in the aftermath of medical harm. ${ }^{1}$ The immediate impetus for this was a recommendation in the public inquiry report into the failings at Mid Staffordshire NHS Foundation Trust, following revelations about poor standards of care and the neglect of patients there between 2005-2009. ${ }^{2}$ Unlike the majority of the 290 recommendations contained in this vast three-volume report which remain unimplemented, the Department of Health swiftly signalled its intent to legislate for

\footnotetext{
* ck16846@bristol.ac.uk.

**oliver.quick@bristol.ac.uk.

1 Reg 20 Health and Social Care Act 2008 (Regulated Activities) Regulations 2014.

2 Recommendation 181 of the Report of the Mid Staffordshire NHS Foundation Trust Public Inquiry (The Stationery Office 2018).
} 
candour. ${ }^{3}$ The end product is the lengthy and complex reg 20 of the Health and Social Care Act 2008 (Regulated Activities) Regulations 2014. Scotland introduced its own version on 1 April 2018, ${ }^{4}$ and similar reforms are likely to follow in Ireland and Northern Ireland based on the recommendations in recent inquiry reports. ${ }^{5}$ In Wales, regulations for dealing with complaints and concerns require patients or their representatives to be advised of medical harm, but only in the event of a complaint being made. ${ }^{6}$ Calls for a more wide-ranging legal duty have since been made there ${ }^{7}$ and form part of the Welsh government's legislative programme for 2018-2019. ${ }^{8}$

Being honest with patients who have been harmed by healthcare treatment is a relatively basic requirement, but one which has been problematic in practice. This article examines the new legal duty of candour in its historical context and offers some insight into cultural aspects of medicine that need to be addressed for the new duty to be effective. We first trace the background to the duty, which included an interesting medicopolitical debate about the necessity of turning to law, and also a somewhat unedifying discussion about the level of patient harm which should trigger the duty. ${ }^{9}$ We then provide a detailed analysis of the duty and consider some of the early evidence about its enforcement. In terms of potential obstacles to its effectiveness, our analysis will foreground theories explaining resistance based in practitioners' and healthcare institutions' fear of litigation and prosecution in the UK. However, as we will discuss in our third section, opposition to some forms of candour emerged within the medical profession prior to the emergence of modern liability systems. This paper will argue that to create a culture of candour it is important to look beyond the more commonly identified concerns about litigation and understand these historical trends.

\section{The problem of patient safety and open disclosure}

Ensuring and improving the safety of patient care is a global public health problem. International studies have demonstrated that between 8-12 per cent of hospitalised patients in advanced healthcare systems experience an adverse event whilst receiving care. ${ }^{10}$ Medical error is reported to be a leading cause of mortality in first-world health systems. ${ }^{11}$ The broader category of unsafe care has been estimated to account for 43 million injuries across the world each year. ${ }^{12}$ Whilst the accuracy of such striking claims

3 Department of Health, Patients First and Foremost: The Initial Government Response to the Report of the Mid Staffordshire NHS Foundation Trust Public Inquiry (CM 8576 2013) 46.

4 The Duty of Candour Procedure (Scotland) Regulations 2018 <http://www.legislation.gov.uk/ssi/2018/57/made>.

5 Recommendations 34 and 35 of the Scoping Inquiry into the Cervical Check Screening Programme (Final Report 2018) <http://scallyreview.ie/wp-content/uploads/2018/09/Scoping-Inquiry-into-CervicalCheck-FinalReport.pdf >; and Recommendation 1 of the Report of the Inquiry into Hyponatraemia related Deaths (IHRDNI 2018) <www.ihrdni.org>

6 The National Health Service (Concerns, Complaints and Redress Arrangements) (Wales) Regulations 2011, reg 12(7).

7 K Evans, A Review of Concerns (Complaints) Handling in NHS Wales: 'Using the Gift of Complaints' (Health in Wales 2014) Recommendation 49.

8 <https://beta.gov.wales/legislative-programme $>$.

9 D Dalton and N Williams, Building a Culture of Candour: A Review of the Threshold for the Duty of Candour and of the Incentives for Care Organisations to be Candid (Royal College of Surgeons 2014).

10 C Vincent, Patient Safety (2nd edn, Elsevier 2010) 54.

11 M A Makary and M Daniel, 'Medical Error - The Third Leading Cause of Death in the US' (2016) 353 British Medical Journal i2139.

12 A K Jha, I Larizgoitia, C Audera-Lopez et al, 'The Global Burden of Unsafe Medical Care: Analytic Modelling of Observational Studies' (2013) 22 BMJ Quality and Safety 809. 
is likely to be contestable, the harms associated with patient safety failures are undeniable. Most obviously, there are physical and emotional harms to patients and professionals, but also financial costs to health systems; in 2017-2018, the English NHS Litigation Authority paid out $£ 2.23$ billion in compensation and fees for clinical negligence claims. ${ }^{13}$ From the perspective of patients, such harms may be exacerbated by the secondary harm of being denied a truthful explanation of events. A recent survey of 728 claimants in England confirms that obtaining an explanation and apology continues to motivate the vast majority of clinical negligence claims. ${ }^{14}$ The psychological harm of discovering dishonesty can have a particularly profound impact on patients in terms of trust in professionals and providers of care. ${ }^{15}$

Ensuring and improving patient safety is not easy, involving complex issues of care, competency, culture and communication. ${ }^{16}$ Confronting medical failure and being candid with patients is emotionally difficult for clinicians, ${ }^{17}$ although writing about this has become a popular publishing genre in recent years. ${ }^{18}$ Candour is commonly defined as the 'quality of being open and honest' and is perceived positively. ${ }^{19}$ Arguably, candour is a particularly detailed form of honesty which includes revealing insider information to those who are less well informed. In the context of patient safety, being candid has implications for therapeutic relationships between patients and professionals, but also for organisations in terms of creating the conditions whereby staff feel able and supported to be candid about medical harm. For clinicians, a broad approach to candour extends beyond admitting individual mistakes to informing patients about problems associated with the resourcing and management of hospitals and health systems. This clearly raises issues for clinicians who may feel conflicted in terms of loyalty to their patients, colleagues and the health service which employs them, although professional guidance is clear that patient protection must be prioritised. ${ }^{20}$

Interestingly, codes of medical ethics, such as the Hippocratic Oath and the Declaration of Geneva do not oblige truthfulness. ${ }^{21}$ However, the ethical case for doctors to disclose errors to patients is clear and based on the importance of truth-telling and respect for persons. ${ }^{22}$ Despite support for the principle of disclosure, this has not always translated into practical implementation. In 2005, a National Audit Office report revealed that only 24 per cent of English hospital trusts routinely informed patients who had been victims of adverse incidents. ${ }^{23}$ Research from the USA has suggested a disclosure rate of

13 NHS Resolution, Annual Report and Accounts 2017-18 (2018) 17.

14 Behavioural Insights into Patient Motivation to Make a Claim for Clinical Negligence: Final Report by the Behavioural Insights Team (NHS Resolution 2018) 45.

15 J Robinson, 'The Price of Deceit: The Reflections of an Advocate' in M M Rosenthal, L Mulcahy and S Lloyd Bostock (eds), Medical Mishaps: Pieces of the Puzzle (Open University Press, 1999) 246-56 ; W Powell, 'Robbie's Law: Lack of Candour - The Impact on Patients and their Families' Clinical Risk (3 February 2014).

16 O Quick, Regulating Patient Safety: The End of Professional Dominance (Cambridge University Press 2017).

17 D Hilfiker, 'Facing our Mistakes' (1984) 310 New England Journal of Medicine 118.

18 For example, A Gawande, Complications: A Surgeon's Notes on an Imperfect Science (Profile Books 2003); H Marsh, Do No Harm: Stories of Life, Death and Brain Surgery (Weidenfeld \& Nicolson 2014); S Westaby, Fragile Lives: A Heart Surgeon's Stories of Life and Death on the Operating Table (Harper Collins 2018).

19 Oxford English Dictionary.

20 GMC, Raising and Acting on Concerns about Patient Safety (GMC 2012) para 10.

21 T L Beauchamp and J F Childress, Principles of Medical Ethics (5th edn, Oxford University Press 2001) 283.

22 N Berlinger, After Harm: Medical Error and the Ethics of Forgiveness (John Hopkins University Press 2005); MLSmith and H P Forster, 'Morally Managing Medical Mistakes' (2000) 9 Cambridge Quarterly of Healthcare Ethics 38.

23 National Audit Office, A Safer Place for Patients: Learning to Improve Patient Safety (The Stationery Office 2005) 4. 
between 30 per cent and 40 per cent. ${ }^{24}$ This evidence also suggests that doctors are less likely to disclose when errors are not obvious to patients, or when they have more serious consequences. Regrettably, disclosure has often been half-hearted and based on halftruths. ${ }^{25}$ Ultimately, the principled commitment to candour has not prevented what Leape and Berwick have called an 'ethically embarrassing debate' within healthcare about whether or not to disclose such harmful events. ${ }^{26}$

A complex range of factors conspire to explain this 'disclosure gap'. Fundamentally, this is explained by a system of medical education which has not prioritised the communication skills necessary for effective disclosure. ${ }^{27}$ Fear about a range of repercussions, including the threat of legal and disciplinary actions, is also commonly cited as a factor inhibiting disclosure. In the healthcare context, professionals worry about the legal significance of saying too much, despite the legislative promise in England and Wales that 'an apology, an offer of treatment or other redress, shall not of itself amount to an admission of negligence or breach of statutory duty'. ${ }^{28}$ Survey research from the USA and Australia confirms that healthcare professionals state that the fear of law is the main barrier to the practice of open disclosure. ${ }^{29}$ This is consistent with a vast body of literature which considers the barriers to reporting of adverse incidents in medicine. In a comprehensive analysis of research published between 1980-2014 into barriers to incident reporting, Archer et al produced a useful synthesis of the available evidence. The authors found that the three most frequently cited barriers to incident reporting were: 'fear of adverse consequences including litigation (161/748), process and systems of reporting (110/748) and incident characteristics $(92 / 748) \cdot 30$ We return to a discussion of this research in section 3 below, but turn now to a review of recent policy responses to the problem of encouraging open disclosure to patients.

Concerns about a lack of openness and the chilling effect of law prompted health systems around the world to introduce policies aimed at encouraging clinicians to be open with harmed patients and their carers. Australia introduced the first national Open Disclosure standard in 2003, which has been updated into a national framework and is overseen by the Australian Commission on Safety and Quality in Health Care. ${ }^{31}$ There

24 R J Blendon et al, 'Views of Practicing Physicians and the Public on Medical Errors' (2002) 347 (24) New England Journal of Medicine 1933; L Lopez et al, 'Disclosure of Hospital Adverse Events and its Association with Patients' Ratings of the Quality of Care’ (2009) 169 (20) Quality of Care Archives Internal Medicine 1888.

25 E O'Connor et al, 'Disclosure of Patient Safety Incidents: A Comprehensive Review' (2010) 22(5) International Journal for Quality in Health Care 371.

26 L Leape, and D Berwick, 'Five Years after To Err Is Human: What Have We learned? (18 May 2005) 293(19) Journal of the American Medical Association 2384, 2388.

27 L Leape et al, Unmet Needs: Teaching Physicians to Provide Safe Patient Care (Report of the Lucian Leape Institute Roundtable on Reforming Medical Education, National Patient Safety Foundation 2010).

28 S 2 of the Compensation Act 2006.

29 T H Gallagher et al, 'Patients' and Physicians' Attitudes Regarding the Disclosure of Medical Errors' (2003) 289 Journal of the American Medical Association 1001; D Studdert et al, 'Legal Aspects of Open Disclosure II: Attitudes of Health Professionals - Findings from a National Survey’ (2010) 193(6) Medical Journal of Australia 351.

30 S Archer, L Hull, T Soukup et al, 'Development of a Theoretical Framework of Factors Affecting Patient Safety Incident Reporting: A Theoretical Review of the Literature' (2017) 7 BMJ Open doi:10.1136/bmjopen2017-017155.

31 Australian Commission on Safety and Quality in Health Care, Australian Open Disclosure Framework (ACSQHC 2013). 
have been similar initiatives in New Zealand, ${ }^{32}$ Canada $^{33}$ and Ireland. ${ }^{34}$ In England, the now defunct National Patient Safety Agency issued a best practice framework about 'Being Open' in 2009. 35 This was supported by statements encouraging openness by the NHS Litigation Authority ${ }^{36}$ (now rebranded as NHS Resolution) and also enshrined in the NHS Constitution, ${ }^{37}$ which pledges to:

... ensure that when mistakes happen or if you are harmed while receiving health care you receive an appropriate explanation and apology, delivered with sensitivity and recognition of the trauma you have experienced, and know that lessons will be learned to help avoid a similar incident occurring again.

Whilst all laudable attempts at encouraging openness and honesty, these policy initiatives and pledges are legally unenforceable. They also lack evidence about their effectiveness in actually altering behaviour. ${ }^{38}$ The creation of the statutory duty of candour is arguably a reflection of the failure of such policies for affecting meaningful change. However, before analysing this duty, it is worth remembering that the possibility of placing candour on a legal footing has long been considered. For example, in Lee v South West Thames Regional Health Authority ${ }^{39}$ Sir John Donaldson MR stated that 'some thought should be given to what is the duty of disclosure owed by a doctor and a hospital to a patient after treatment', albeit that this issue wasn't central to the appeal in that case. Two years later, in the case of Naylor $v$ Preston ${ }^{40}$ the same judge went further in stating that in professional negligence cases, and in particular in medical negligence cases, there is a duty of candour resting on the professional man'. Whilst the common law hasn't evolved to create such a duty, the idea of so doing should not really be considered radical. The duty of care in negligence endorses the prudent patient test for determining the standard of care in relation to information disclosure before medical intervention, ${ }^{41}$ so why should the same not apply to post-treatment communication?

Although previous calls for creating a legal duty of candour had been unsuccessful, ${ }^{42}$ a combination of campaigning and the catalyst of a high-profile public inquiry report prompted governmental action. The immediate trigger was provided by recommendation 181 in the Francis report into the Mid Staffordshire NHS Foundation Trust Public Inquiry. This called for a statutory duty of candour on healthcare providers and registered healthcare professionals who believe or suspect that treatment or care has caused death or serious injury. ${ }^{43}$ As we see below, the enacted duty is different in two important respects: it is limited in its application to organisations, but broader in applying to

32 Health and Disability Commissioner, Guidance on Open Disclosure Policies (HDC 2009).

33 Canadian Patient Safety Institute, Canadian Disclosure Guidelines. Being Open with Patients and Families (Canadian Patient Safety Institute 2011).

34 Health Service Executive, Open Disclosure Policy (HSE 2013).

35 National Patient Safety Agency, Being Open: Communicating Patient Safety Incidents with Patients, their Families and Carers (NPSA 2009).

36 NHS Litigation Authority, Saying Sorry (NHS Litigation Authority 2017).

37 The NHS Constitution for England (2015) (Complaint and Redress: Your Rights).

38 O Quick, 'A Scoping Study on the Effects of Health Professional Regulation on those Regulated' (Council for Healthcare Regulatory Excellence 2011).

39 [1985] 1 WLR 845, 851.

40 [1987] 1 WLR 958, 967.

41 Confirmed by the UK Supreme Court in Montgomery v Lanarkshire Health Board [2015] UKSC 11.

42 Ian Kennedy (Chair), The Report of the Public Inquiry into Children's Heart Surgery at the Bristol Royal Infirmary 19841995: Learning from Bristol (Cmnd 5207 (I) 2001); Department of Health, Making Amends: A Consultation Paper Setting out Proposals for Reforming the Approach to Clinical Negligence in the NHS (Department of Health 2003).

43 Recommendation 181 of the Report of the Mid Staffordshire Inquiry (n 2). 
moderate harm, as well as to death or serious injury. The longer-term explanation must be credited to the campaigning of Mr William Powell aided by the medical charity Action against Medical Accidents (AvMA). Mr Powell's son Robbie died in 1990 aged nine from undiagnosed Addison's disease, a rare auto-immune disorder of the adrenal glands. Whilst the health authority admitted liability in relation to the failure to diagnose and made a payment of $£ 80,000$, in Powell $v$ Boladr ${ }^{44}$ an action for psychiatric harm suffered by his parents, the Court of Appeal held that no duty of care existed between the doctor and Robbie's parents. Regrettably, the court held that the doctors were not legally obliged to explain the circumstances surrounding the death of Robbie to his parents, thus further illustrating tort law's ambivalent relationship with patient safety. ${ }^{45}$

Whilst the General Medical Council (GMC) responded by revising its guidelines to oblige candour in such circumstances, ${ }^{46}$ the option of creating a separate legal duty met with considerable medical resistance. For example, a statement by the Medical Defence Union, ${ }^{47}$ in response to the House of Commons Health Committee recommendation for introducing a statutory duty of candour in 2009, is revealing and worth quoting in full:

We do not support the Committee's recommendation that the Chief Medical Officer's proposal for a statutory duty of candour be considered. The inference of the recommendation is that no effective duty of candour currently exists, but this is not the case for doctors who already have an ethical duty and our experience is that doctors do raise concerns. We do not know what the sanction would be if such a legal duty were introduced, but doctors can already be erased from the medical register if their fitness to practise is impaired because they have not complied with GMC guidance. Surely that is sanction enough?

This statement was problematic in several respects. The claim that an ethical duty of candour is effective is inconsistent with the research evidence noted above, which suggests a relatively low rate of disclosure to patients. In terms of the medical profession, relying on the GMC to enforce the ethical duty also appears to be somewhat misplaced. A parliamentary debate in 2010 revealed that the GMC had not dealt with a single case for breach of the duty contained it its guidance. ${ }^{48}$ Nevertheless, despite the lack of enforcement activity around candour, professional regulators have demonstrated their commitment to candour in the form of revised guidance. ${ }^{49}$ It is worth noting that the duty enshrined in professional regulation is broader than the legal duty in applying to situations where something has gone wrong with 'treatment or care causes, or has the potential to cause, harm or distress' ${ }^{50}$ Whilst this guidance was a welcome regulatory reaffirmation of the importance of being open, it was not enough to prevent the creation of a statutory duty of candour to which we now turn to analyse.

44 (1998) 39 BMLR 35.

45 J Miola, 'The Tort of Negligence and Patient Safety' in J Tingle and P Bark (eds), Patient Safety, Law Policy and Practice (Routledge 2011).

46 GMC, Good Medical Practice (GMC 1998).

47 Medical Defence Union, press release 3 July 2009.

48 HC Deb 1 December 2010, cols 276WH.

49 Professional Standards Authority, Can Professional Regulation Do More to Encourage Professionals to be Candid when Healthcare or Social Work Goes Wrong? Advice to the Secretary of State for Health (Professional Standards Authority 2013); General Medical Council and Nursing and Midwifery Council (NMC), Openness and Honesty when Things Go Wrong: the Professional Duty of Candour (GMC/NMC 2015).

50 GMC/NMC (n 49). 


\section{The statutory duty of candour}

The statutory duty of candour is contained in reg 20 of the Health and Social Care Act 2008 (Regulated Activities) Regulations 2014. Reflecting the style of much modern-day statutory drafting, this is a lengthy provision which requires careful reading. ${ }^{51} \mathrm{It}$ is striking that, whilst telling harmed patients the truth may seem a basic requirement, this has been translated into legal complexity via nine detailed clauses within reg 20. It came into force in November 2014 and initially applied only to 'health service bodies' which means health and social care organisations registered with the CQC. Primary care organisations, dentists, private healthcare and adult social services were initially excluded, but brought within reg 20 from April 2015, albeit with a different harm threshold for triggering the duty. The duty applies in England and to organisations rather than individual healthcare professionals, who are instead bound by the ethical duties of candour within professional codes of conduct noted above. However, in practice it is expected that those responsible for the patient's care would be expected to have candid conversations with harmed patients, although the regulation refers to 'representatives' of the organisation, who may therefore not be clinicians.

There are two parts to the statutory duty. First, reg 20(1) imposes a general requirement for 'registered persons' to be open and transparent with patients or their representatives about care and treatment. This reflects the aim of creating a culture of candour which is seen as crucial to improving patient safety. In the words of the influential Williams and Dalton report which argued strongly in favour of the duty: 'A culture of candour is a culture of safety, and vice-versa. 52 Second, there are specific reporting requirements placed on providers in relation to 'notifiable safety incidents'. This is set out in reg 20(2) and involves notifying, apologising and supporting patients who have suffered harm as a result of such an incident. This includes providing them with an honest account of the facts about the incident, advice on appropriate further enquiries and must be followed up by written notification (reg 20(3) and (4)).

A key issue which dominated pre-legislative debate was in relation to setting the appropriate harm threshold for triggering the duty. Many who opposed the duty then argued in favour of confining it to cases involving death or severe harm. The argument that honesty should depend on the degree of harm suffered by patients was unprincipled and ultimately rejected by a Department of Health-commissioned review of the evidence and competing arguments. In a strongly worded report, ${ }^{53}$ Professor Sir Norman Williams (then President of the Royal College of Surgeons) and Sir David Dalton, Chief Executive of an NHS trust, recommended that candour be extended to cases of moderate harm, where the bar has now been set. In fact, the term moderate is itself slightly misleading given that it includes significant harm such as 'unplanned return to surgery, an unplanned readmission, a prolonged episode of care, extra time in hospital or as an outpatient, cancelling of treatment, or transfer to another treatment area' (reg 20(7)). The duty does not apply to harm deemed to fall below moderate, or to near misses, with legitimate fears about the bureaucratic burden and defensive documentation around candour defeating this. ${ }^{54}$

51 J R Spencer, ‘The Drafting of Criminal Justice Legislation - Need it be so Impenetrable?’ [2008] Criminal Law Journal 585; D Greenberg, 'Dangerous Trends in Modern Legislation’ (2015) (Jan) Public Law 96.

52 Dalton and Williams (n 9) 12.

53 Ibid.

54 D Berwick, A Promise to Learn - A Commitment to Act (National Advisory Group on the Safety of Patients in England August 2013) 34. 
Regrettably, 'notifiable safety incident' is defined differently depending on whether care was provided by 'health service bodies' (organisations registered with the CQC such as hospital trusts) or 'other registered persons' (primary care organisations, dentists, private healthcare organisations and adult social services). In relation to 'health service bodies', notifiable safety incidents are defined as 'any unintended or unexpected incident that occurred in respect of a service user during the provision of a regulated activity that, in the reasonable opinion of a healthcare professional, could result in, or appears to have resulted in' death, severe, moderate or prolonged psychological harm to the service user (para 8). In terms of primary care organisations, dentists, private healthcare organisations and adult social services, there is no requirement for them to inform patients about incidents which 'could' result in significant harm but haven't yet done so (reg 20(9)). According to the CQC, the 'definitions have been differentiated in this way to account for the different notification systems for health service bodies and all other providers. In doing so, they are intended to reduce the administrative burden caused by the introduction of this new statutory duty of candour. 55 Whilst concerns about the additional workload associated with delivering candour are valid, it is nevertheless regrettable that the commitment to openness is potentially diluted in this context. For the duty to be effective in creating a culture of candour, it is imperative that all signs point in the same direction. Having such a distinction effectively permits a weaker form of candour outside of NHS secondary healthcare.

The statutory duty is enforced by the CQC, the health and social care regulator in England, as one of 13 'fundamental standards of care' it monitors. The CQC has no specific approach to monitoring compliance with the duty, but approaches it as part of its inspection of whether good care is being provided. ${ }^{56}$ It claims to focus on whether systems are in place to deliver greater candour, as opposed to looking for specific breaches of the duty, although surely the latter would be good evidence of whether the system itself is effective. It has stated that such systems would include training and supporting staff to communicate with patients about safety incidents, and reporting systems which might include the recording of the duty of candour notification. 57 Regulatory action includes refusal or removal of registration, issuing warnings, special measures, fines and prosecution. Whilst prosecutions are likely to be rare, it is worth noting that breaches of the duty may be prosecuted without a warning notice being issued by the CQC. It is disappointing that the CQC has no statistics in relation to the implementation of a fundamental standard of care and no central training programme for enforcement. The lack of centralised records is a concern, although the CQC has been able to confirm that 15 actions have been taken against NHS trusts and 90 actions taken against primary care and private care providers in relation to failure to comply with the duty. ${ }^{58}$ These are mainly in the form of 'requirement notices', for example, to provide adequate training to staff about the duty. Overall, the early evidence suggests a light-touch approach to regulating the duty, with concerns raised about a lack of detailed reference to candour in CQC inspection reports.

The bureaucratic process of delivering and monitoring candour risks losing sight of its grounding in ethical values. With this in mind, the Williams and Dalton review warned that:

55 CQC, Regulation 20: Duty of Candour. Information for all Providers: NHS Bodies, Adult Social Care, Primary Medical and Dental Care, and Independent Healthcare (CQC 2015) 9.

56 Ibid 7.

57 Ibid 10.

58 H Blythe, Regulating the Duty of Candour: A Report by Action against Medical Accidents on CQC Inspection Reports and Regulation of the Duty of Candour (AvMA 2015). 
A compliance-focused approach will fail. If organisations do not start from the simple recognition that candour is the right thing to do, systems and processes can only serve to structure a regulatory conversation about compliance. The commitment to candour has to be about values and it has to be rooted in genuine engagement of staff, building on their own professional duties and their personal commitment to their patients. ${ }^{59}$

Whether a compliance approach to candour will fail in practice remains to be seen. These are still early days for the legal duty and there is a need for research evidence to understand its positive and negative impacts. There is some limited anecdotal evidence that the duty has led to increased disclosure to patients and empowered staff to remind colleagues about the legal obligation. ${ }^{60}$ For those who opposed the duty, such as medical defence organisations, the reliance on law and regulation was misconceived and might even exacerbate existing disinclinations to disclose. Without denying the possible negative impact of legal and regulatory interventions, it is important to understand that the tension between candour and concealment in medicine has been a persistent theme in medical practice for hundreds of years. Our next section will explain the existence of tensions around this issue long before litigation and insurance cultures could have contributed to the creation of a professional norm.

\section{Lessons from history: cultures of truth-telling in medicine 1800-1950}

As described above, Archer et al's recent comprehensive review of scholarship on the issue of disclosure of medical errors drew on work produced across relevant disciplines and methodologies, incorporating quantitative, qualitative and mixed-methods studies. It is our hypothesis that the findings of these modern empirical studies of medicine can be enhanced by also considering the works of historians of medicine and the medical profession on this issue. This will enable us to more fully understand the professional and cultural context in which any aversion or inclination to candour developed, prior to the emergence of insurance and litigation cultures in medicine.

This section considers debates over candour in medicine across the period 1800 to 1950. In the early nineteenth century, the medical profession in the UK took on a form recognisable today with the emergence of, what is often termed in historical accounts, 'hospital medicine'. ${ }^{61}$ It is generally seen as a period of increasing professionalisation in medicine which was accompanied by important regulatory developments such as the first moves toward accreditation for practice and the concept of 'legally qualified practitioners' in the Apothecaries Act 1815.62 It was also the period in which the forerunner to the British Medical Association was founded in 1832 as a professional interest group for medical doctors, by the middle decades of the century playing an active role in the regulation of the practice of medicine, via the Medical Act of 1858, which established the GMC. ${ }^{63}$ Equally of importance for this inquiry, during the nineteenth century the

59 Dalton and Williams (n 9) 17.

60 P Walsh, 'Challenges and Opportunities for Patient Safety and Justice in the UK' (2018) 23(1) Journal of Patient Safety and Risk Management 7; J McHale, 'Patient Safety, The "Safe Space" and the Duty of Candour: Reconciling the Irreconcilable?' in J Tingle, C O Neill and M Shimwell (eds), Global Patient Safety: Law, Policy and Practice (Routledge 2018).

61 On 'hospital medicine', see E H Ackerknecht, Medicine at the Paris Hospital, 1794-1848 (Johns Hopkins University Press 1967).

62 I Loudon, Medical Care and the General Practitioner, 1750-1850 (Oxford University Press 1986).

63 Harry Eckstein, Pressure Group Politics: The Case of the British Medical Association (Allen \& Unwin 1960); M J D Roberts, 'The Politics of Professionalization: MPs, Medical Men, and the 1858 Medical Act' (2009) Medical History 53. 
practice of medicine became more akin to the medicine that is practised today. Hospital medicine, or 'modern medicine' emerged in Britain in the early 1800s and represented a revolutionary change, moving as it did away from the intimate relationship between practitioner and patient at the bedside, drawing on Galenic or constitutional theories that were specific to each patient and their own narrative of illness. ${ }^{64}$ Histories of medicine that consider the issue of candour focus on either traditions of truthful reporting of failure to other doctors, or (less commonly) traditions of truth-telling to patients.

Early pioneers of modern approaches to medicine were hugely excited by the potential 'hospital medicine' presented for collating, refining and disseminating the results of medical experiments and treatments. Often this collectivising project in the service of improving medicine necessitated the reporting of failure. War was an important driver of this idea and the Napoleonic Wars (1803-1815) cultivated medical administrators such as Dr James McGrigor, Surgeon-General for the Duke of Wellington's army in Spain and Portugal during the Peninsular Wars (1808-14) and later Head of the British Army Medical Department, who used his powerful position heading a vast department treating thousands of sick and injured men to institute cultures of reporting among medical officers. ${ }^{65}$ Statistics were collected and mined for useful data, but McGrigor also encouraged (and sometimes demanded) what we would now call case notes of treatments, both successful and failed, with the specific purpose of finding out what was effective or not and disseminating that knowledge throughout his department. For this to work, in a time when medicine was arguably 'less certain', it was important that failure was well documented. Thus, in the vast military medical enterprises of the early to mid-nineteenth century, strong cultures of reporting failure were developed. McGrigor's confidence in the importance of candid reporting to other practitioners is borne out in his published account of the Army Medical Department's work on the Egyptian Campaign in 1801:

Humble as the labours may seem, and confined as the abilities of an individual may be, were he only faithfully to relate observations made with care, to compare them with those of his contemporaries, and by these to correct the opinions of his predecessors, he would perform no mean service to his art. ${ }^{66}$

Regularised returns and reports encouraged the candid discussion of failed experiments or attempts to combat disease, and encouraged a culture of openness regarding medical practice. Alongside this open culture, military norms allowed senior officers to push for regularisation of practice and the close surveillance of practitioners who were considered to be using treatments differing widely from consensus approaches:

It is very desirable that an eye be kept on the cases of Hospital Gangrene which have been treated . . . by SS Burmeister, and that the final result of each be known. This mode of treatment is at variance with that which has ultimately been found successful in most of the Hospitals in the Peninsula. ${ }^{67}$

64 On 'neo-classical' medicine, see, N Jewson, 'Medical Knowledge and the Patronage System in Eighteenth Century England' (1974) 8 Sociology 369; for studies discussing the evolution of medicine in Britain at this time, see: R French and A Wear, British Medicine in an Age of Reform (Routledge 1991); C Hamlin, Public Health and Social Justice in the Age of Chadwick, Britain 1800-1854 (Cambridge University Press 1998); C Lawrence, Medicine and the Making of Modern Britain 1700-1920 (Routledge 1994); I Loudon, 'Medical Practitioners 1750 1850 and the Period of Medical Reform in Britain' in A Wear (ed), Medicine in Society (Cambridge University Press 1992).

65 C Kelly, War and the Militarization of British Army Medicine 1793-1830 (Pickering \& Chatto 2011) esp chs 3 and 5.

$66 \mathrm{~J}$ McGrigor, Medical Sketches of the Expedition from Egypt to India (London 1804) 56-7.

67 Wellcome Library, Royal Army Medical Corps Collection, 799/6 (book II) 229: McGrigor to D I Higgins, 28 January 1814. 
While failure was something about which there was a culture of 'candour' in an intraprofessional sense within the military, those failures were also communicated to civilian medical audiences - for example, McGrigor published in medical journals about the successes and failures of military medicine. This replicated an existing culture in civilian medicine of case reports - often detailing failed interventions - in journals such as MedicoChirurgical Transactions. A simple example of one of the hundreds of such reports to be found is as follows from the Edinburgh Medical and Surgical Journal:

I. Case of Brachial Aneurism - Mr W.G. of East Aytoun, near Scarborough, was bled in the median basilic about the 20th October 1835, and unfortunately the point of the lancet penetrated the brachial artery. From the blood flowing per saltum it was apprehended that such was the case, and, in order to effect a cure, compression was employed, but without any effect, as in three weeks, a pulsating tumour, of the size of a hen's egg had formed at the elbow, bearing the usual cases of aneurism. ${ }^{68}$

In civilian medicine at this time, most surgery was performed in the presence of an audience, literally in a theatre. Thus, questions of candour or concealment of mistakes may have been moot, contributing to a broader culture of intra-professional candour. The public display of failure, however, was not without consequence. Botched operations, or perceptions of medical incompetence, could elicit much comment and debate in the pages of medical journals such as The Lancet and could even permeate the popular press. ${ }^{69}$

In a detailed study of mistakes in civilian medicine, 'Learning from Mistakes: Early Twentieth Century Surgical Practice 1900-1920', ${ }^{\circ}$ Wilde and Hurst consider the work of Archibald Watson, an Australian doctor who had been educated in Gottingen and Paris, was a Fellow of the Royal College of Surgeons of England, and professor of anatomy at the University of Adelaide. He made an extraordinary contribution through his observation and note-taking on thousands of surgical operations in Australia, as well as in South Africa, the USA and in Britain. He used his notes in teaching, and one student recalled that he would 'illustrate his subject with unqualified descriptions of the surgical triumphs and disasters' he had witnessed. ${ }^{71}$ Watson's focus was on difficult or 'elite' surgery, but nonetheless by the sheer volume of cases he witnessed, his records enable the modern reader to glean a strong sense of surgical practices and cultures at the time. Watson criticised others but was also very critical of himself. Wilde and Hirst argue that his diaries are good evidence that many 'adverse events' we experience now - such as postoperative bleeding after tonsillectomy - were also experienced by early twentiethcentury surgeons and patients. Watson also recorded many professional arguments about the best way to perform operations, which, as Wilde and Hirst explain, demonstrates the difficult tension present when investigating 'failure' - it can be hard to draw the line between a clear error and a practice which was a matter for legitimate debate. Watson's journals reveal that debates over best practice were often carried on loudly among the medical audience of operations at the Mayo Clinic. As Wilde and Hirst point out 'Doing things the "right way", and finding better ways to do things, were overlapping categories;

68 'Report of Various Surgical Cases' (1839) 140 EMSJ 139.

69 For an example, see discussion of the case of Bransby Cooper in M Brown, "Bats, Rats and Barristers": The Lancet, Libel and the Radical Stylistics of Early Nineteenth-century English Medicine' (2014) 39 Social History 182.

70 (2009) 64 Journal of the History of Medicine and Allied Sciences 38.

71 Ibid 46. 
but it is often difficult or impossible to draw any distinction at all between doing things the wrong way and failed attempts to find a better way to perform an operation. ${ }^{72}$

Watson was well known by his peers for his critical observation of surgical operations and his pull-no-punches style. However, it is clear from Wilde and Hirst's study that his candour was not resented by his colleagues, or the surgeons he observed. On the contrary, Wilde and Hirst show that the profession embraced Watson's searing honesty - in 1899 he gave a paper to the gynaecology and obstetrics division of the Australian Medical Congress, entitled 'The Saving of Blood in Gyneaecological Operations'. His lecture drew heavily on his records of mistakes, and what not to do, from his notebooks. The lecture was received with a strong positive reaction, indeed doctors in the room 'fell over themselves to pay tribute' to him and his contribution to their art. From this we can surmise that at least in an 'intra-professional' context the majority of surgeons were open to the importance of discerning and learning from mistakes. Importantly, however, Watson did not publish what he saw; his observations and critiques were passed on by word of mouth. In the small number of cases where Watson did identify a fatal technical error being made, 'none of those responsible was called to account to anyone except their immediate peers and their consciences' ${ }^{73}$

The notion that observation of failure was important, but to be kept strictly within the profession, is reinforced by other historical studies such as Sally Wilde's work on the diaries of Sydney urologists, John Laidley and Malcolm Earlam, and their study trips to Britain and North America in the 1930s and 1940s. Based on these diaries, Wilde concludes that different forms of candour were considered important and that some forms of concealment were considered highly unethical and punished within professional networks and structures: 'evidence of dishonesty was treated with a shrug and a metaphorical "cold shoulder", while evidence of honesty about bad results was greeted with warm approbation'. ${ }^{74}$ Importantly, however, these sanctions were still very much held within the professional sanctum. Similarly, histories detailing the work of Harvey Cushing, the pioneering neurosurgeon, emphasise his practice of writing up case notes and publicising his errors in the hope of educating others. ${ }^{75}$ The American Surgeon Ernest Codman took the concept further and developed what he called the 'end-resultsystem', based on the notion 'that every hospital should follow every patient it treats long enough to determine whether or not the treatment has been successful and if not, ask "why not?" with a view to preventing similar failures. ${ }^{76}$

These historical studies build a picture of a medical profession which embraced the importance of intra-professional candour for the improvement of medicine; based on peer observation, recording of failure and following up on patients. An important distinction is drawn out by the professional reaction to Codman, who advocated public reporting of deficiencies in medical work - including an assessment of reasons for the failure of the treatment. Unlike Watson, he faced some considerable opposition. By replacing individual conscience with documented competence as a basis for clinical evaluation, Codman was thought to be challenging the moral and professional autonomy

72 Ibid 52 .

73 Ibid 58 .

74 S Wilde, The History of Surgery: Trust, Patient Autonomy, Medical Dominance and Australian Surgery 1890-1940 (Finesse Press 2011) 132.

75 R L Pinkus 'Mistakes as a Social Construct: An Historical Approach' (2001) 11(2) Kennedy Institute of Ethics Journal 117; M Bliss, Harvey Cushing: A Life in Surgery (Oxford University Press 2005).

76 E A Codman (1984) xii, quoted in V A Sharpe and A I Faden, Medical Harm: Historical, Conceptual and Ethical Dimensions of Iatrogenic Illness (Cambridge University Press 1998) 29. 
of the physician. However, despite its unpopularity with many doctors, his work gained credibility in 1916 when the American College of Surgeons incorporated his Committee on Hospital Standardization, an attempt to study and improve hospital outcomes, and it was his work which led to early moves towards accreditation and hospital quality measures in the USA in the 1950s.

In contrast to the reasonably well-settled professional view on intra-professional candour described above, the question of whether doctors should be candid with their patients has always been more fraught. In a more recent study Gallagher et al reported that:

Physicians agreed that harmful errors should be disclosed but 'choose their words carefully' when telling patients about errors. Although physicians disclosed the adverse event, they often avoided stating that an error occurred, why the error happened, or how recurrences would be prevented. Patients also desired emotional support from physicians following errors, including an apology. However, physicians worried that an apology might create legal liability. ${ }^{77}$

However, it is questionable whether legal liability on its own provides the central plank of professional resistance to candour to patients at the turn of the nineteenth century. As Wilde describes:

... when things went wrong there was an inclination from the courts and from the press to trust that those concerned did their best, and there was an almost fatalistic acceptance of poor outcomes. Surgeons were seldom called to account for themselves before either the court of public opinion, or via civil litigation. ${ }^{78}$

Despite the low risk of litigation, professional anxiety about candour to patients was exemplified at this time through commentary in Frederick Treves' Manual of Operating Surgery 1892, 'the leaving of a sponge or instrument within the peritoneal cavity is a catastrophe which no surgeon would feel greatly disposed to make public'. ${ }^{79}$ Claire Brock suggests this is indicative of the 'level of secrecy surrounding such incidents' within the professional culture of the time, ${ }^{80}$ but it may be that she draws too long a bow, and instead this quote is more solid evidence of an acknowledgment of the devastating impact of such a revelation on the professional reputation (and thus livelihood) of the doctor.

The issue of how doctors approached telling patients about errors historically can be difficult to glean - it is not common for historical actors to leave extensive evidence of secrets and concealment. The sources above give us insight into the anxieties of the profession, but much of what we know of historical traditions of candour to patients in medicine or debates about its importance comes from studies of consent to treatment and, so, often relate to candour prior to treatment and focus on the experience of the patient. Much of the literature on consent builds on or responds to Jay Katz's seminal work The Silent World of Doctor and Patient in which he claimed that 'disclosure and consent, except in the most rudimentary fashion, are obligations alien to medical thinking and practice'. ${ }^{81}$

A more sympathetic perspective on the development of candour-led dialogue between doctor and patient is advanced by Andreas-Holger Maehle, who coins a useful term in his research on doctor-patient dialogues and questions of consent and

77 T H Gallagher, A D Waterman et al, 'Patients' and Physicians' Attitudes regarding the Disclosure of Medical Errors' (2003) 289(8) Journal of the American Medical Association 1001.

78 Wilde (n 74) 33

79 F Treves, Manual of Operating Surgery vol II (Cassell \& Company 1892) 234.

80 Claire Brock, 'Risk, Responsibility and Surgery in the 1890s and Early 1900s' (2013) 57(3) Medical History $317,322$.

81 Jay Katz, The Silent World of Doctor and Patient (The Free Press 1984) 1. 
truthfulness when he refers to 'medical traditions of truth-telling'. He points to long-held ideas about the constitution and emotional state of the patient, and the notion that doctors should not 'alarm and harm' those in fragile conditions. While families of patients might be given a more candid assessment of the patient's condition and prognosis, 'restricted truth telling' was often seen to be in the best interest of the patient throughout the eighteenth and nineteenth centuries. ${ }^{82}$ This attitude was reflected in Percival's Statement of Medical Ethics published in 1803 in which it was stated that there were cases where telling the truth might even kill the patient and in that circumstance 'the practitioner shall sacrifice that delicate sense of veracity . . to this claim of professional justice and social duty'. ${ }^{83}$

In another study of this issue which pushes back against the trope that candour is alien to the medical profession, 'Truth, Trust, and Confidence in Surgery', Sally Wilde considered questions of candour and consent in the context of the 'dramatic rise in the range of surgical procedures that doctors ... were prepared to attempt' 84 during a period of intense surgical innovation and development at the beginning of the twentieth century. Along with this rise in the number of procedures was a dramatic rise in the number of people who were prepared to undergo them. In this piece Wilde challenges the received view that patients lacked autonomy in this period and, importantly, that doctors didn't care about it. She argues that consent and trust, especially from private paying patients, was won through communication between doctors, patients, their families and friends. Her study considers published case reports in the 1890s from two hospitals (one in London, one in Brisbane, Australia). She notes in this period (mirroring medical print culture described in the 1800 s above) that doctors publicised their successful operations, as well as those ending in death, and suggests that this was a way of publicly explaining or making excuses for failures. She argues there were many cases where consent was given, even from some public patients who admittedly had less autonomy than their feepaying counterparts. While consent was often recorded, records of provision of information to patients about the dangers of the operation are rare but quite often present where the operation was particularly dangerous, exploratory or experimental. ${ }^{85}$ In addition she highlights the scrutiny surgeons were under from coroners' courts, the press, their peers and also from patients who sued or refused to pay when they didn't like the outcome. Instead of building a culture centred on secrecy and litigation avoidance, in Australia this prompted a consensus view that full disclosure of risks prior to treatment was the best way for doctors to protect themselves and, Wilde argues, also bred a culture of trust between doctor and patient more generally.

Moving forward in time we continue to see historical studies that emphasise the nuanced cultures of truth-telling in medicine, and which problematise the professional, cultural and reputational aspects of professional hesitation. In his seminal 1970 study, Forgive and Remember: Managing Medical Failure, Charles L Bosk reported on his investigation of a major teaching hospital in America. ${ }^{86}$ This is one of the only studies of this time that directly looked at errors and the practicalities of self-regulation in surgery. Mirroring the

82 A H Maehle, 'Silence or Negotiation? Doctor Patient Dialogue and the Questions of Consent and Truth in Late Nineteenth Century England' Social History of Medicine Conference, Liverpool 2018.

83 T Percival, Medical Ethics: Or, a Code of Institutes and Precepts, Adapted to the Professional Conduct of Physicians and Surgeons (S Russell 1803) 166.

84 S Wilde, 'Truth, Trust, and Confidence in Surgery, 1890-1910' (2009) 83 Bulletin of the History of Medicine 302-330, 302-3.

85 Ibid 315.

86 (University of Chicago Press 1970). 
observations of Wilde in the nineteenth century, Bosk noted that doctors perceived strong distinctions between technical errors and breaches of the 'moral code of surgery' - or what he termed normative errors. Echoing the attitude of his historical forbears, technical errors were perceived as inevitable and provided learning opportunities attracting only minor penalties, if punished at all. Normative errors, including dishonesty and the hiding of mistakes, were perceived as more serious - reflecting on the character and trustworthiness of the doctor concerned and, in Bosk's observation, not often forgiven.

Cumulatively, the historical studies considered above show that the medical profession has long embraced the clinical importance of learning from mistakes and thus favoured intra-professional candour. However, it is also apparent that the profession has not been united on the need for an equivalent approach to patients.

\section{Conclusion}

It is an easy reflex to turn to the effects of insurance requirements and the zero-sum game of adversarial litigation as explanations for the emergence of non-candid cultures in medicine today. However, it is unlikely that, historically, those are the only reasons for the emergence of a professional culture which doesn't seem to fit with candour. It may well be that notions of what 'failure' is and what it means (or does not mean) about expertise, about the dynamic of trust and power within the therapeutic relationship, and about the relationship of the medical profession with the public have as much to do with it. All the historical studies that touch on the question of candour from doctors to patients highlight complexities in the medical profession's attitude. While it appears that informal professional sanction and disapprobation could be, and often was, seen as an appropriate response to any dishonest behaviour, such as actively hiding mistakes, there has traditionally been very little support for a norm requiring candour about mistakes to patients. While cultures of truth-telling have been important and perceived as important to the mission of medicine for several hundred years, historically, it appears that the central debate for doctors, even in the absence of insurance and litigation, was not whether it was important to observe, reflect and honestly discuss failure, but whether it was necessary (or helpful) to do so with patients. One of our concerns about the duty of candour is that its legal complexity could erode the historically strong commitment of practitioners to openness amongst themselves about failure as a cooperative learning project in medicine.

The duty of candour as enacted seems a blunt and confusing instrument with which to unpick the various barriers for medical professionals identified in this article. Although there are early signs that the duty has provided some support to professionals who already have a strong commitment to the principle, the confusing, convoluted and highly legalistic and fragmented construction of the duty creates another obstacle for any health practitioner already intimidated by the potential consequences of a candid disclosure. Even the far-reaching and onerous connotations of the word 'candour' itself have the potential to put off the uncertain practitioner considering disclosing an adverse event. The enduring problem of defining medical failure is likely to be played out again in terms of agreeing the boundaries of notifiable safety incidents, especially in terms of what is appropriately deemed 'unexpected'. The recent decision in Montgomery $v$ Lanarkshire Health Board places significant emphasis on ongoing and engaged dialogue between patient and clinician. ${ }^{87}$ It may be that a similar approach to discussion of adverse events within the complex system of a healthcare episode, rather than a laser focus on the single incidence

87 [2015] UKSC 11, [2015] 2 WLR 768. 
of an individual's failure, could provide healthcare workers with a more accessible way to contextualise an incident and engage in a conversation about it with affected patients and their families. For the duty to work, its operation and requirements need to be clearer, less legally impenetrable, and give more reassurance to healthcare workers about the relationship between a candid disclosure and legal liability. Regulators need to consider the profession's historical commitment to reducing failure through intra-professional candour in contrast to its aversion for candour with patients. Such attitudes reflect an unsurprisingly surgical need for empirical evidence of therapeutic benefit to motivate disclosure and could signal strategies more likely to attract professional approbation. 